\title{
A Portable Capillary Electrometer
}

This content has been downloaded from IOPscience. Please scroll down to see the full text. 1902 Proc. Phys. Soc. London 18377

(http://iopscience.iop.org/1478-7814/18/1/334)

View the table of contents for this issue, or go to the journal homepage for more

Download details:

IP Address: 129.78.139.28

This content was downloaded on 02/10/2015 at 22:45

Please note that terms and conditions apply. 
XXXIV. A Portable Capillary Electrometer. By S. W. J. Smite, M.A., Demonstrator in Pkysics, Royal College of Science, London.*

\section{[Plate V.]}

TnIs instrument is a modification of the form of capillary electrometer represented in the first figure, and consisting of two wide tubes joined across by a capillary tube which is cylindrical, and may be horizontal or may slope upwards

Fig, 1.

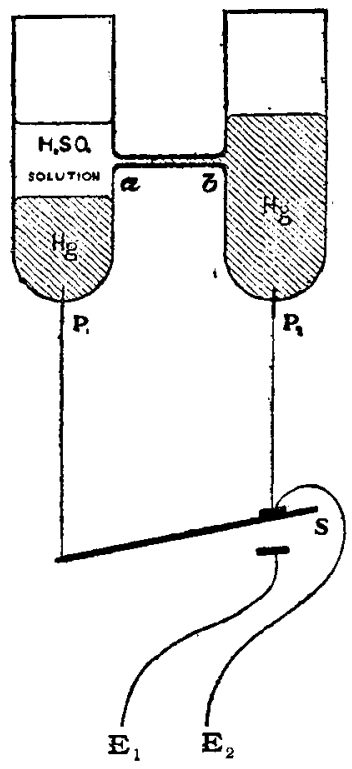

at any angle from $b$ towards $a$. The apparatus contains mercury and sulphuric acid of about maximum conductivity distributed roughly as shown. A spring-key, like that represented in the figure, is commonly used with the instrument, and keeps the platinum terminals $P_{1}$ and $P_{2}$ at the same potential unless the lever $\mathrm{S}$ is depressed. When the lever is depressed the potentials of the terminals become $E_{1}$ and

* Read December 12, 1902.

VoL. $\mathrm{x}$ VIII. 
$\mathrm{E}_{2}$, which may, for example, be the potentials of two points in a potentiometer circuit. It is the function of the instrument to determine whether these potentials are the same or different.

The nature of the modifications made in the present electrometer is shown in the second figure.

To prevent evaporation of the sulphuric acid solution, without preventing free motion of the liquids within the apparatus, the wide tubes are closed at the top; but are joined across by another tube opening into them as shown. With this arrangement the apparatus is made air-tight, and can, if desired, be made air-free by exhaustion of the apparatus before sealing. It is obvious also that the apparatus can be upset without spilling of the liquid. The two limbs are of equal size, and the capillary passes from the middle of one to the middle of the other. The apparatus contains approximately enough mercury to completely fill one limb, and about half as much sulphuric acid solution. By suitably adjusting the distribution of the mercury and the solution in the two limbs, the apparatus can be arranged for use with the capillary tube either borizontal or tilted upwards at a considerable angle. The maximum angle of tilt available is increased by increasing the lengths of the limbs in comparison with the length of the capillary. It is easy to construct an electrometer which can be used with the capillary at almost any inclination between horizontal and vertical.

The distribution of the mercury can be altered most easily by means of a cross-piece provided with a tap, as represented in the figure by dotted lines. When the tap is open there is free communication between the mercury in the two limbs, and the relative amount in each can be altered by tilting the apparatus. When the tap is closed the two quantities of mercury are insulated from each other. The addition of this arrangement tends to complicate the construction of the instrument, and, although it is very convenient in practice, it is not indispensable. Any desired changes in the distribution of the mercury and the solution can usually be effected without much trouble, by means of the capillary and the upper cross-tube.

To prevent the platinum wires forming the electrodes being wetted by the acid solution, if the apparatus should acci- 
dentally, or during transit, be laid on its side or turned upside down, the lower ends of the tubes may be drawn out, as shown at $P_{1}^{\prime}$ and $P_{2}^{\prime}$ in the second figure, and slightly constricted above the ends of the platinum terminals. This precaution may be dispensed with if the electrodes are formed by welding pieces of platinum foil, which are afterwards amalgamated, on to the ends of the wires $P_{1}$ and $P_{2}$. The apparatus can then be turned upside down and shaken, so that the amalgamated foil becomes wetted by the acid, without any further ill-effect being produced than an occasional slight wandering of the zero of the instrument for a short time after it is righted again.

The usual spring-key has several disadvantiges. Thus, if it is made of brass, the contacts frequently become unsatisfactory through surface tarnishing, and if, to avoid this, the hearing surfaces are made of platinum the key sometimes shows pronounced thermoelectric effects. Further-and this is a point of some importance in a portable instrument-the key cannot conveniently be fastened on to the same stand as the rest of the instrument, for, unless the stand and the support on which it rests are very rigid, the pressure necessary to depress the spring produces sufficient movement of the meniscus, by change in the inclination of the capillary tube during the act of depression, to render the detection of minute changes of surface-tension impossible.

The mercury-key, represented in the second figure, is free from these disadvantages. It consists, as shown, of a $U$-tube closed at one end and communicating at the other with a pneumatic-pressure ball and containing mercury in the bend. Three platinum wires are fused into the tube and connected as shown. It is obvious that the same change of contacts is produced by squeezing the ball $B$, as by depressing the lever $\mathrm{S}$ in the spring-key. The mercury in this key takes the place of the lever in the spring-key, and the two different contacts between it and $\mathrm{E}_{1}$ and $\mathrm{E}_{2}$ respectively are h're quite definite and practically independent of the amount of pressure exerted upon the ball B. Further, the contacts are not exposed directly to the laboratory atmosphere. The thermoelectric effects are very small since the changing contacts take place between platinum and mercury which are almost identical thermoelectrically. The warmth com- 
municated to the key from the hand of the operator can bo neglected, and the heat produced by the compression of the air in the key need only be very small. The key can be fixed to the same stand as the rest of the apparatus, for even if the pressure which changes the contacts is applied as suddenly as possible the maximum vertical pressure upon the stand is only a few grams, whereas in the case of the ordinary spring-key it may be 500 grams or more.

By adjusting the length of the mercury column in the key so that it is only slightly less than the length of the U-tube between the two extreme platinum wires, the change of potential at $P_{1}$ from $E_{2}$ to $E_{1}$ and conversely, can be made almost instantaneously. Hence measurements can be made with the instrument even if the zero is altering fairly rapidly. With a key of this kind it is also obvious that the observation of the meniscus through the microscope can receive a very near approach to undivided attention.

Commutators and lieys which make a set of connexions in a prescribed order can be constructed on the same principle as the pneumatic ley above described. The third figure represents a combined commutator and electrometer key.

Fig. 3.

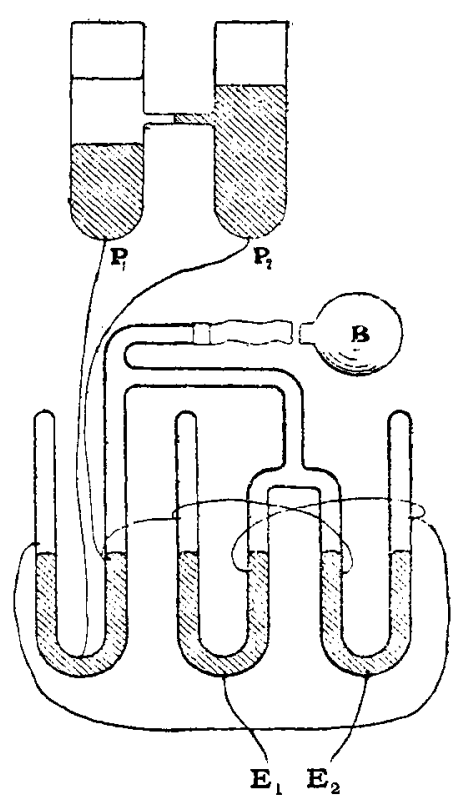

The U-tube to the left is the key already described, and the double $U_{-t u b e}$ to the right is the commutator. The positions of the different wires are so arranged with respect to the levels of the mercury, that it requires greater pressure to change the contacts in the double $U$-tube, than in the tube to the left. The first effect of pressure is to throw the electrometer into the potentiometer or other circuit, connecting $\mathrm{P}_{1}$ with $\mathrm{E}_{1}$ and $\mathrm{P}_{2}$ with $\mathrm{E}_{2}$. On increasing the pressure, the connexions in the double $U$-tube are reversed and $P_{1}$ is con. 
nocted with $E_{2}$ and $P_{2}$ with $E_{1}$. Hence, when the contacts change in the commutator, we get a motion of the electrometer meniscus corresponding to approximately twice the difference of potential between $\mathrm{E}_{1}$ and $\mathrm{E}_{2}$, and so, in this way, the sensitiveness of the instrument is doubled.

While it is clear that to get the minimum thermoelectric effect in mercury-platinum keys, it would be necessary to use a method of changing the contacts in which compression of air in the key does not take place, yet the thermoelectric electromotive forces which occur in the pneumatic keys described are much too small to produce an observable effect upon the readings of the most sensitive capillary electrometer*.

The sensitiveness of the electrometer, using the simple key first described, is such that when the diameter of the wide tubes is about $1 \mathrm{~cm}$. and the diameter of the capillary is about $1 \mathrm{~mm}$., a movement of the meniscus perceptible with certainty in a microscope magnifying 100 times is produced by a potential-difference equal to 0001 volt. The actual extent of the movement is somewhat variable, and amounts usually to about $01 \mathrm{~mm}$. The folloring numbers, obtained with apparatus previously described (Phil. Trans. A. 1899, vol. cxciii. p. 63), show the effect of comparatively large polarizing electromotive forces upon the surface-tension between mercury and sulphuric acid solution of density $1 \cdot 17:-$

E.M.F. Volts.
$\mathbf{0}$
$\mathbf{0 . 0 2 0 2}$
$\mathbf{0 . 0 4 0 4}$
$\mathbf{0 . 0 6 0 5}$
$\mathbf{0 . 0 8 0 7}$
0.1009

Surface-Tension.
$\gamma$
$1.021 \gamma$
$1.040 \gamma$
$1.059 \gamma$
$1.080 \gamma$
$1.097 \gamma$

$\gamma$ is the "natural" surface-tension between the mercury and the solution. Its approximate value is $300 \mathrm{ergs}$ per sq. $\mathrm{cm}$. Judging from these numbers, it would seem that the surfacetension is altered by about one part in 10,000 by a polarizing

* Since this paper was read I have found that mercury keys (similar in principle to those referved to above) in which the thermoelectric effects are reduced to a minimum are described by Kamerlingh Onues, Leyden Communications, No. 27, p. 31, 1896. 
E.M.F. of $\cdot 0001$ volt. In the case in which the capillarytube is horizontal and the wide tubes are vertical, the relation between the motion of the meniscus $\delta x$ and the corresponding change in surface-tension $\delta \gamma$ is expressed approximately by

$$
a^{2} \rho g \delta x=\mathrm{A} c \delta \gamma
$$

if we assume that the capillary and wide tubes are of uniform cross-section- $a$ being the area of cross-section of the capillary, $c$ its circumference, A the area of cross-section of the wide tubes, and $\rho$ the sum of the densities of the mercury and the solution. From this formula it would appear possible to produce a much larger movement of the meniscus than $.01 \mathrm{~mm}$. by means of a potential-difference of 0001 volt by using a very narrow capillary, especially if the crosssection of the capillary were elliptical instead of circular. It is found in practice, however, that the motion of the meniscus under minute polarizing forces is controlled very largely by stickiness and accompanying changes in the angle of contact between the liquids and the glass, and by variation in the cross-section of the tube at the place where the meniscus rests. The sensitiveness of the instrument to small electromotive forces is practically as great when the diameter of the capillary is $1 \mathrm{~mm}$. as when it is very much less. A very narrow capillary is less easy to manipulate than a comparatively wide one (about $1 \mathrm{~mm}$. in diameter) and is only advantageous when it is desirable that the capacity of the instrument should be as small as possible, or that its action should be as rapid as possible. In the latter case the length of the capillary should also be small.

If the capillary-tube, instead of being horizontal, is inclined at an angle $\theta$ to the horizontal, the equation given above becomes

$$
a g\left\{\left(\rho_{1}+\rho_{2}\right) a / \mathrm{A} \cdot \cos \theta+\left(\rho_{1}-\rho_{2}\right) \sin \theta\right\} \delta x=c \delta \gamma,
$$

in which $\rho_{1}$ is the density of the mercury, and $\rho_{2}$ that of the solution. From this equation it follows: (1) that if $\theta$ is positive (mercury thread sloping upwards towards the meniscus) the sensitiveness is not increased appreciably by making $A$ very large in comparison with $a$, unless $\theta$ is very small; and (2) that if $\theta$ is negative (meniscus at lowest part of thread) the mereury becomes unstable when $\theta$ is numerically greater than $\tan ^{-1}\left(\rho_{1}+\rho_{2}\right) a /\left(\rho_{1}-\rho_{2}\right) A$, i. e. when $\theta$ is numerically 
greater than a/A approximately, since in order that the polarization may be practically confined to the capillary electrode, the ratio $a / \mathrm{A}$ must be small. It also follows that if it were proposed to design an instrument which should have the utmost sensitiveness possible, attention would have to be paid to the straightness of the capillary as well as to the uniformity of its cross-section. It is not necessary, however, to take the precautions here indicated in order to obtain the sensitiveness already quoted; it may be obtained with almost any capillary-tube chosen at random, and with $\theta$ having such a positive value that the restoring force for a small displacement is considerable enough to render the instrument easy to work with.

The sensitiveness of 0001 volt, which is obtainable without any difficulty if the mercury is clean, is sufficient for a great many measurements in which the electrometer can be employed, and for these the electrometer (which for the purpose in question is really a surface-tension galvanometer) is more convenient than an ordinary galvanometer with a suspended magnetic system. The electrometer is much more easily set up than an ordinary galvanometer. There is no suspension, no lamp and scale, and, practically, no levelling; but it is advisable when working with the instrument that the potential of $E_{1}$ should never be more than a few tenths of a volt less, or more than about a volt greater, than the potential of $E_{2}$. These conditions of working are, in general, not difficult to satisfy. If the potential-differences applied exceed either of the limits above mentioned, it is snmetimes necessary to run some of the mercury through the capillary tube in order to get the instrument again into its best working order. The capillary electrometer is already used almost universally in physico-chemical laboratories, and the modifications herein suggested may, perhaps, have the effect of slightly extending the sphere of its usefulness. The accompanying Plate V., taken from a photograph, represents one form of the instrument described. Its size is about two-fiftbs of that of the actual instrument. The arrangement of the different parts will be obvious from the description already given. The illumination of the end of the mercury-thread is effected by means of a concave mirror attuched to the base of the instrument. 


\section{Discussron.}

Dr. R. T. GlazeBroor congratulated the author, and said that workers with the capillary electrometer would appreciate the improvements introduced. He said that the author had given the minimum E.M.F. which the instrument could detect, and asked what was the maximum E.M.F. which could be safely applied. He referred to the advantages of the mercury keys exhibited, and remarked that, by their use, many difficulties were obviated.

Dr. LAETELDT said the mercury keys were improvements on those used by Prof. Onnes. In these keys the contacts were changed by the motion of mercury brought about by tilting. The tubes containing the mercury were exhausted to prevent oxidation.

Mr. SMITH, in reply to Dr. Glazebrook, said that, roughly, the maximum E.M.F. should not exceed balf a volt.

XXXV. Note on an Elementary Treatment of Conducting Networks. By L. R. Wilberforce, M.A., Professor of Physics at University College, Liverpool*.

IT may be worth while to point out that the well-known reciprocal relations between the parts of a conducting network can be readily established without an appeal to the properties of determinants.

Let $A, B, C, D, \ldots$, be a number of points connected by conductors $A B, A C, A D, \ldots, B C, B D, \ldots, C D, \ldots$, of resistances $R_{A B}, \ldots$, and suppose that currents $Q_{A}, \ldots$, are led into the network at the points $A, \ldots$, from without, subject to the condition $Q_{A}+Q_{B}+\ldots=0$, and that internal electromotive forces, $\mathrm{E}_{\mathrm{AB}}, \ldots$, act in the conductors in the directions $A B, \ldots$ Let the currents in the conductors be $C_{A B}, \ldots$, and let the potentials at $A, \ldots$, be $V_{A}, \ldots$ The fact that there is no continuous accumulation of electricity at any point gives us a series of equations whose type is :-

$$
\mathrm{QA}_{\mathrm{A}}=\mathrm{C}_{\mathrm{AB}}+\mathrm{C}_{\mathrm{AC}}+\ldots . . \cdot \text {. . . }
$$

and the application of Ohm's law gives us a series whose type is

$$
\begin{gathered}
\mathrm{R}_{\mathrm{AB}} \mathrm{C}_{\mathrm{AB}}=\mathrm{V}_{\mathrm{A}}-\mathrm{V}_{\mathrm{B}}+\mathrm{E}_{\mathrm{AB}} . \quad . \quad \cdot \quad \cdot . \\
\text { * Read January } 28,1003 .
\end{gathered}
$$




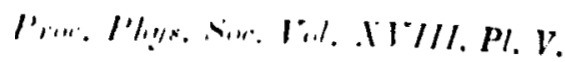

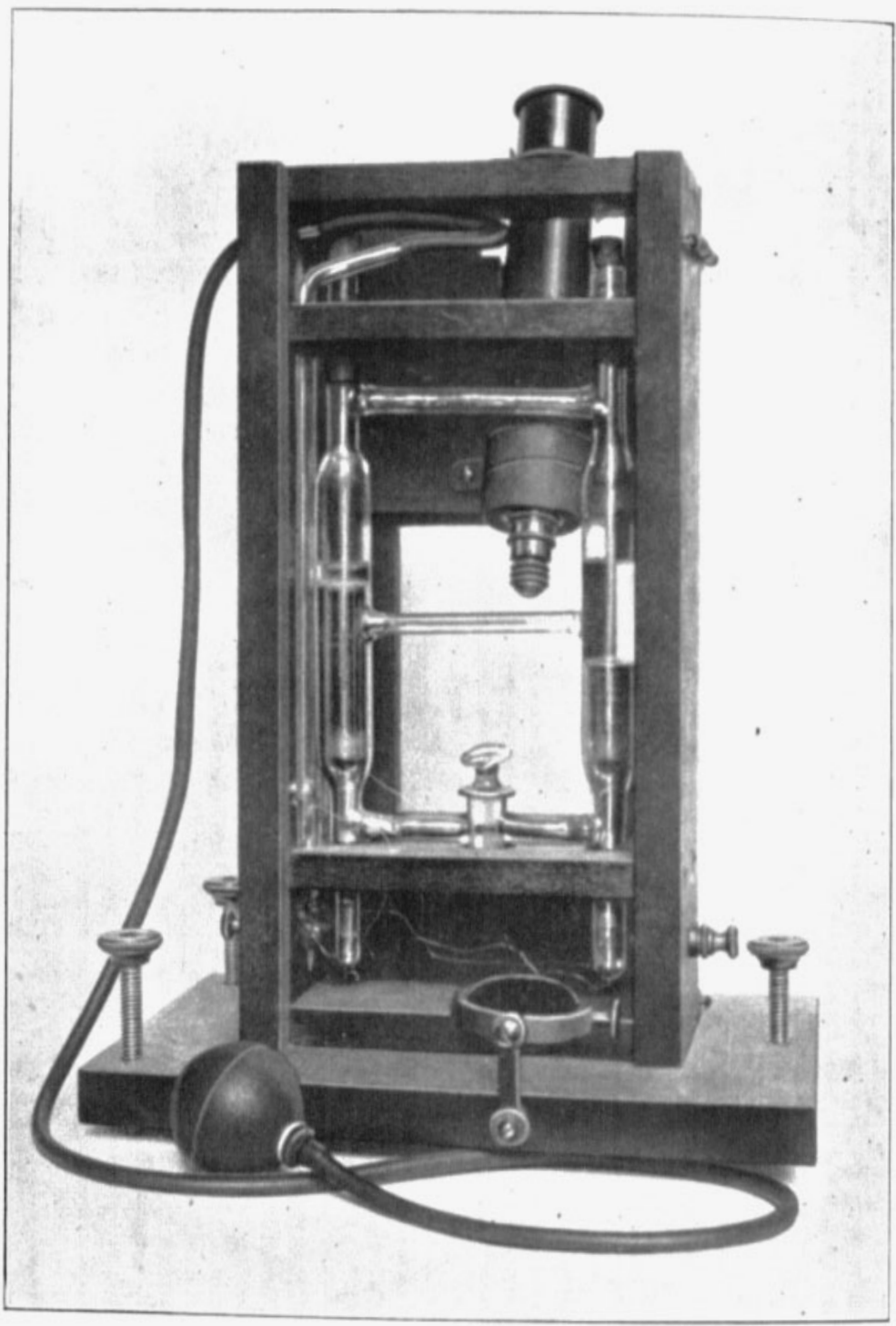

